\title{
ATM TRANSPORT BETWEEN UMTS BASE STATIONS AND CONTROLLERS: SUPPORTING TOPOLOGY AND DIMENSIONING DECISIONS
}

\author{
Ana-Belén García, Manuel Alvarez-Campana, Enrique Vázquez, Guillermo Guénon, Julio Berrocal
}

Dept. of Telematics - Technical University of Madrid (DIT-UPM), Ciudad Universitaria s/n, 28040 Madrid, Spain, \{abgarcia, mac, enrique, guenon, berrocal\}@dit.upm.es

\begin{abstract}
The access transport infrastructure of a cellular communications network represents a significant proportion of the total network cost. This prevents the network designer from over-dimensioning these links. In the case of UMTS (Universal Mobile Telecommunications System) access network, the use of a packet-switched technology (ATM for the first Release of the system) and the multi-service nature of the traffic to be carried rise the dimensioning process complexity. At this respect, we have developed a simulation tool for supporting the network designer when making dimensioning and physical topology decisions related to Iub interface (between each UMTS base station and its controller), which is the most critical interface of UMTS access network. This simulator is capable of modelling the presence of multiple traffic classes as well as the Iub transport protocol stack with enough accuracy; it offers a graphical configuration interface and has been designed to have a great flexibility when choosing topologies and ATM configurations.
\end{abstract}

Keywords - UMTS Access Network, ATM, Network Simulation, Quality of Service, Traffic Characterization.

\section{INTRODUCTION}

Capacity resources have become cheaper with time, especially in large core networks, in which sometimes it is more convenient to provide over-dimensioned links than to introduce complex Quality-of-Service (QoS) mechanisms into the nodes.

Access networks, however, can still be network segments with serious capacity constraints. For instance, if we think of a cellular communications network, it is clear that the most cost-effective solution is not to over-dimension the thousands of access links an operator can manage. Instead, careful dimensioning and design is needed in order to optimize the infrastructures cost.

The problem becomes even more difficult if we are facing a packet-switched transport technology that must be able to adequately carry an open set of services with tight QoS constraints. This is precisely the case of the UMTS (Universal Mobile Telecommunications System) interface between each Node-B (base station) and its associated RNC (Radio Network Controller), the so-called Iub interface. The transport technology specified for lub interface in UMTS
Release 99 specifications is ATM. (Note that from Release 5 on, IP is also proposed as a transport alternative to ATM in the access network. We have only considered the current ATM option in this study.)

With our work we aim to provide a useful tool that supports the UMTS-network operator when facing the topology and dimensioning decisions regarding Iub interface. As stated above, this is a complex problem which also includes many degrees of freedom. This is why we have chosen to develop a simulation model, instead of using analytical models, in order to include all the most significant low-level details with an adequate precision.

\section{IUB TOPOLOGY AND DIMENSIONING ISSUES}

UTRAN (UMTS Terrestrial Radio Access Network) terrestrial protocols are structured in two layers: Radio Network Layer (RNL), consisting of all the UMTS-specific protocols (including radio protocols), and Transport Network Layer (TNL), which includes generic protocols, and is in charge of conveying RNL data across the terrestrial interfaces with the necessary QoS. In our study we consider the Iub transport infrastructure dimensioning, thus the significant radio protocols effects will be included as part of the sources behavior.

As we can see in Fig. 1, AAL2 (ATM Adaptation Layer 2) and ATM are the protocols used in the TNL User Plane of the lub interface. The physical layer is the one that best fits the operator's needs (e.g. E1 lines).

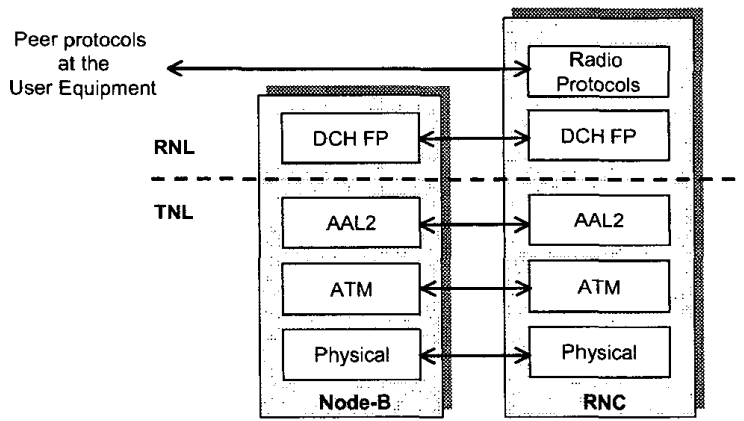

Fig. 1. User-plane Iub protocol stack for dedicated channels.

Although, from a logical perspective, the lub interface is a point to point link between the Node-B and its RNC, 
nothing in the UMTS specifications prevents the operator from implementing any other physical infrastructure (see [1] and [2]). For instance, an ATM network with several links and switches can be used to implement the Iub section or part of it. Even if an ATM network is used, there can also be geographical zones in which it is convenient to arrange several Node-Bs directly in a chain or tree configuration, with its corresponding traffic concentration properties. In our study, the scenarios with an ATM network have been given priority, since this can be a quite usual situation for UMTS operators with pre-existing ATM infrastructure.

In summary, when designing the transport network of Iub interface, the operator has different important decisions to make, among them the following: kind and capacity of physical interfaces (conditioned in part by equipment constraints), ATM service category to use for each traffic class (when there is an ATM network with policy function activated), bandwidth to be provided for each user group or concentration and multiplexing strategies. We have developed our tool with all these factors in mind, in order to provide the operator with a means for comparing different strategies and help it to adopt the most convenient dimensioning decisions. The next section is devoted to describing this simulation model.

There are a number of recent investigation results related to UMTS (and specifically UTRAN) QoS provisioning. To name a few, in [3] an UMTS network simulator is presented which is capable of measuring end to end (not only access network) QoS. It is mainly centered on the UMTS-related protocols (e.g. radio protocols) and above. Besides we can find simulation studies about a specific UMTS protocol or set of protocols (see for instance [4] for an analysis of TCP/IP over Radio Link Control layer). There are also papers dedicated to the AAL2/ATM transport in the UTRAN: See in [5] an example of simulation results regarding scheduling mechanisms at AAL2 level, and in [6] an analytical approach to the AAL2/ATM performance analysis with only voice users. We feel that our work is complementary to those just mentioned, in the sense that it directly addresses the particular transport network decisions inside the Iub interface (something not included in the cited papers) with a high level of detail, although not forgetting the most relevant radio protocols characteristics.

\section{DESCRIPTION OF THE TOOL}

\section{A. General overview}

Our simulation tool models the Transport Network Layer of the lub interface. It has been developed to run on a commercial simulation platform, concretely OPNET Modeler [7]. This software gives enough flexibility to create new models (or to adapt existing ones) while providing the developer and the user with a graphical environment to configure simulations and network topologies.
Each Iub simulation scenario comprises three kinds of components, which can be interconnected by ATM links of any capacity:

- Source node: it represents the radio network controller (RNC), since we consider the downlink traffic (presumably the most significant one in typical client-server Internet-like applications). The RNC includes both the traffic sources and the AAL2 and ATM multiplexers.

- Destination node: it models the Node-B, responsible of performing the ATM and AAL2 de-multiplexing, as well as the traffic sink functions (among them, the QoS statistics calculation).

- $\quad$ ATM switch: inside the ATM network, its mission is to model the standard ATM switching of virtual channels (VC) or path (VP) connections.

See the following sections for a detailed description of the most significant aspects of this tool.

\section{B. Variable bit rate sources}

Each individual user session (whether packet switched or circuit switched) in our model represents an active user (for instance, during a telephone conversation) and it makes use of a dedicated transport channel (DCH). The resulting DCH Frame Protocol (DCH FP) frames are carried by an AAL2 connection across Iub. This is consistent with the decision adopted by most UMTS operators for the initial deployment of the service. (Note however that in the future it is possible that the some combination of non-dedicated transport channels is used for bursty data traffic; one example is High Speed Downlink Packet Access, HSDPA, specified as part of the Release 5 of UMTS.)

We have considered that many real applications behave as variable bit rate sources, something important especially when it comes to make an efficient bandwidth assignation, since it allows the user to evaluate the benefits of the statistical multiplexing gain [8]. In the current simulator version each source is equivalent to a two-rate generator, which alternates between High and Low states. These states are directly associated with two allowed rates of the corresponding Radio Access Bearer.

Due to the semi-periodical characteristics of the radio protocols (and also DCH) frame generation, during each of the two defined states (High and Low) each user session generates constant-sized frames, with an interarrival time equal to the TTI (Transmission Timing Interval) of the bearer. Both the sizes and TTI of the frame generation are deduced from the Radio Access Bearer characteristics (see [9]). On the other hand, the statistical characterization of the transit time in each state (High and Low) is something more dependant on the end application characteristics (size of the files the user is downloading, activity factor of speech, etc.). 
The simulation tool allows to define as many groups of identical sources (with the characteristics explained above) as needed. An individual source may generate its traffic with an stochastic offset with respect to the rest of sources inside the same group.

As examples of source parameterizations, in Table 1 we show the attributes chosen for two types of users (namely voice and web users). These two kind of sources are the ones utilized in the simulation studies shown in section IV below.

Table 1. Voice and web traffic parameters.

\begin{tabular}{|c|c|c|}
\hline Application & Voice AMR & Web \\
\hline $\begin{array}{l}\text { Radio Bearer } \\
\text { (kbit/s max. UL/DL) }\end{array}$ & $\begin{array}{c}\text { Conversational, } \\
\text { CS } \\
(12.2 / 12.2)\end{array}$ & $\begin{array}{c}\text { Interactive or } \\
\text { Background, PS } \\
(64 / 64)\end{array}$ \\
\hline $\begin{array}{l}\text { Chosen rates } \\
\text { (kbit/s DL High/Low) }\end{array}$ & $12.2 / 1.95$ & $64 / 0$ \\
\hline $\begin{array}{l}\text { Physical meaning } \\
\text { of High/Low rates }\end{array}$ & $\begin{array}{l}\text { Active speech / } \\
\text { Silence } \\
\text { description }\end{array}$ & $\begin{array}{l}\text { File download / } \\
\text { reading time }\end{array}$ \\
\hline TTI (ms) & 20 & 20 \\
\hline $\begin{array}{l}\text { Frame sizes } \\
\text { (Bytes High/Low) }\end{array}$ & $\begin{array}{l}\text { const }(40) / \\
\text { const }(13)\end{array}$ & $\begin{array}{l}\text { const }(174) / \\
\text { No-frames }\end{array}$ \\
\hline $\begin{array}{l}\text { State duration } \\
\text { (seconds High/Low) }\end{array}$ & $\begin{array}{l}\exp (\text { mean:3) } \\
\exp (\text { mean: } 3)\end{array}$ & 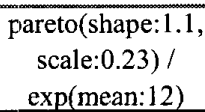 \\
\hline
\end{tabular}

For more information on the physical meaning of these parameters see [10]. Some of the concrete distributions and parameters have been extracted or derived from the recommendations given by $3 \mathrm{GPP}$ inside report [11] regarding simulation studies.

\section{Topology and multiplexing configurations}

When designing the simulator we sought a great flexibility regarding the modelled network topology and the ATM multiplexing strategies.

On one hand, it is possible to include an ATM network and to connect its ATM switches following any physical topology. It is also possible to arrange several Node-Bs in chain (or tree), modelling the internal switching capability that Node-Bs may have, which can be very convenient in some geographical zones. See in Fig. 2 examples of Iub physical configurations of interest in our study.

On the other hand, the user has a great freedom when connecting the different nodes using any combination of ATM VCs and VPs. ATM virtual channels and paths can be of any traffic class (CBR, rt-VBR, nrt-VBR, ABR and UBR) and the traffic sources inside the modelled RNC can use any of them, and are multiplexed at AAL2 level in order to share the VC or VP matching their solicited ATM category.

In order for the ATM categories to have full meaning, the tool is provided with mechanisms to enforce the traffic
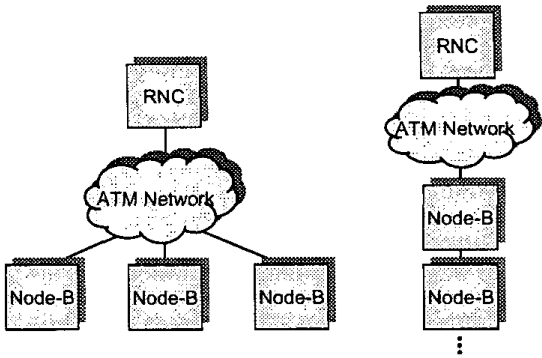

Fig. 2. Examples of Iub physical configurations.

contract: ATM switches on the network can be configured to perform traffic policing (or UPC - Usage Parameter Control) function. UPC, when enabled, will discard cells pertaining to a VP or a VC that are not compliant with the traffic contract. If several ATM virtual channels share the same virtual path, traffic policing will be performed at VP level.

Other remarkable characteristic of the simulator is the full traffic shaping support in the RNC. This traffic management function can be mandatory for the operator if an ATM network with activated UPC is to be traversed from RNC to Node-B, in order to avoid the discarding of cells at network entry point. Similarly to the UPC case, if several ATM VCs share a common VP, traffic shaping is applied at VP level. Otherwise, it acts at VC level.

Traffic shaping is supported for all the traffic classes in which it can be applied: CBR, rt-VBR and nrt-VBR. In the CBR case, the algorithm limits the cell rate to the PCR value of the VP or VC, queuing cells arriving at a higher rate. For VBR traffic classes, the sustained cell rate is set to SCR, allowing bursts of up to MBS cells at PCR rate, and queuing cells that violate these parameters (actually, the implemented algorithm for VBR is the dual leaky bucket as described in [12]). Traffic shaping can be activated or deactivated for each VP or VC (depending on the applicable case) on an individual basis.

Another significant characteristic of the simulator is its ability to represent several traffic multiplexing strategies. It is possible to devote a separate ATM virtual channel to each source group, with no VP multiplexing. This way we may observe statistical multiplexing gain among the sources of each group (multiplexed by AAL2 layer) and also among the virtual channels sharing the links capacity (provided they have variable-rate behaviour). If we find it useful to isolate (i.e. to reserve resources for) groups of virtual channels, we can configure a scenario in which a second level of multiplexing is introduced by means of establishing virtual paths. For instance, a VP could be dedicated to real time traffic source groups and another VP to non real time ones.

Moreover, it is also possible to share the same virtual channel by different source groups, making it possible to 
have ATM payloads which are shared by traffic of different kinds of users. This is a consequence of having a single AAL2 multiplexing function for all the sources using the same virtual channel, and may give rise to capacity savings if the load generated by each group is very low [5].

\section{Offered statistics}

The simulation tool allows for collecting a large amount of traffic and QoS statistics, as stated in the following paragraphs.

Traffic statistics comprise peak traffic rates and sustained traffic rates for ATM cells and source frames collected wherever they have significance (in any link, ATM virtual connection, network node or the global values for the entire network). Traffic rate statistics at source level (i.e. the user of AAL2 level) are available also in a per-group-of-sources basis, identifying each group by the name of the source node and the name for the group inside the node (for instance, "RNC.Mail_users"), both of which can be chosen by the user of the simulator.

Among QoS statistics are loss rates, delays and delay variations for ATM cells and source frames. Again, statistics can be collected at any point in the ATM network where they are significant (for instance, nodes, links or the entire network), and statistics for the source frames are presented individually for each group of sources.

\section{SAMPLE RESULTS}

The main objective of this paper is to provide a clear description of the possibilities that the developed simulation tool offers to a UMTS access network designer, not being our first priority to present a specific and complete set of simulation results.

However, in order to attain a more complete understanding of the simulator, it is also convenient to see at least a summarized set of actual results obtained with it. This is the purpose of the present section.

We considered two kinds (groups) of users, concretely voice (AMR codec) and web, and several quantities of each of them (see Table 2). The traffic generation characteristics for an individual voice or web source have already been specified inside subsection III.B. In all cases the mean source rate of voice traffic represents a $70 \%$ of total mean generated traffic.

Table 2. Users proportions considered in the simulations.

\begin{tabular}{|l|c|c|c|c|}
\hline Nr. of voice users & 30 & 44 & 59 & 73 \\
\hline Nr. of web users & 10 & 16 & 21 & 27 \\
\hline Name & $30 v 10 \mathrm{w}$ & $\mathbf{4 4 v 1 6 w}$ & $\mathbf{5 9 v 2 1 w}$ & $\mathbf{7 3 v 2 7 w}$ \\
\hline
\end{tabular}

In each simulation run the sources of the same group share an ATM virtual channel, and both VCs are multiplexed into a unique ATM virtual path. Two cases have been considered regarding the VP service category, as described next.
- UBR (no traffic shaping and no UPC). This set of simulations is useful for checking "raw" (i.e. with no interference) traffic parameters of the sources at different levels in order to better understand the traffic behaviour and the appropriate bandwidth reservations for each group if other service category is envisioned.

- rt-VBR with VP-level traffic shaping inside the RNC. In this case we searched for a rational set of VBR traffic contract parameters meeting different delay (QoS) constraints for the two groups of sources. Lower parameters values make it possible to accommodate more traffic within the same capacity but at the expense of a possibly unacceptable increase in experienced delays.

The physical topology and link capacities considered for the different scenarios can be seen in Fig. 3. An RNC and a Node-B are interconnected by an ATM network formed by two ATM switches.

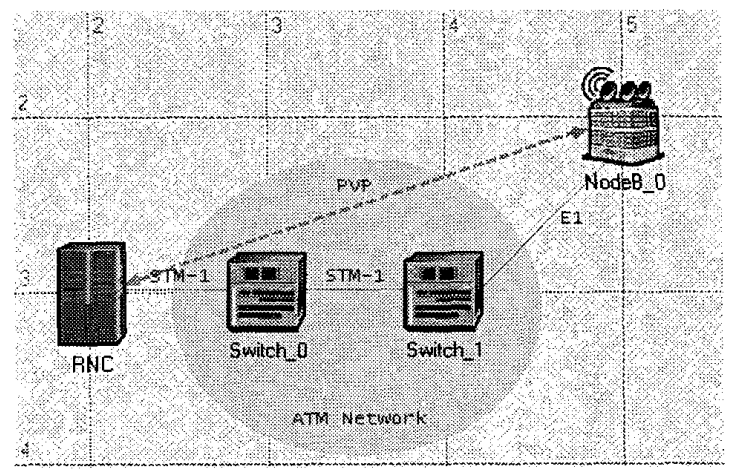

Fig. 3. Physical configuration of the simulation scenarios

Fig. 4 is a sample graph related to the first case mentioned above (UBR VP for all the traffic). It shows ATM peak and mean rates (the statistic is actualized in one-second periods) for voice and web traffic, as well as for the aggregation of both (traffic on the "line"). As we can see, web traffic exhibits a much more bursty behaviour than voice traffic (i.e. mean and peak rates are more different in the web case). Aggregated traffic has also a significant degree of burstiness, which makes it reasonable to try VBR service category if there are important capacity constraints.

Fig. 5, on the other hand, has been obtained from scenarios in which a rt-VBR VP is shared among 44 voice users and 16 web users, with traffic shaping activated. PCR and MBS of the VP have been fixed to $1.98 \mathrm{Mbit} / \mathrm{s}$ (an E1 line rate) and 40 cells, respectively. SCR varied between $10 \%$ and $60 \%$ above the mean ATM rate observed from the previous set of experiments (44v16w combination). In the figure we have represented the percentage of web frames which have exhibited a total delay greater than 5 and 20 milliseconds, as a function of the provisioned SCR of the VP. 


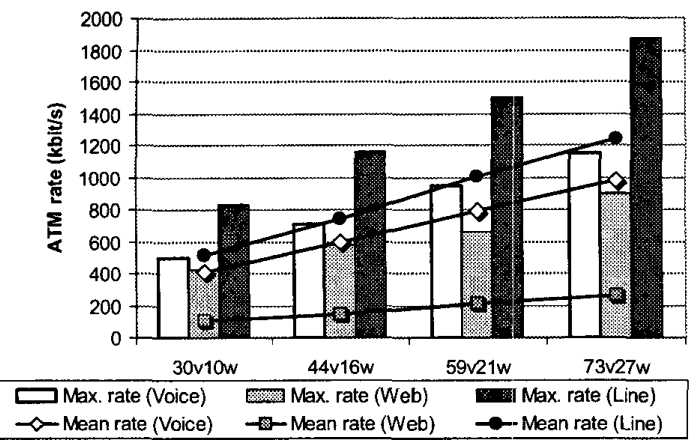

Fig. 4. ATM maximum and mean rates with UBR PVP.

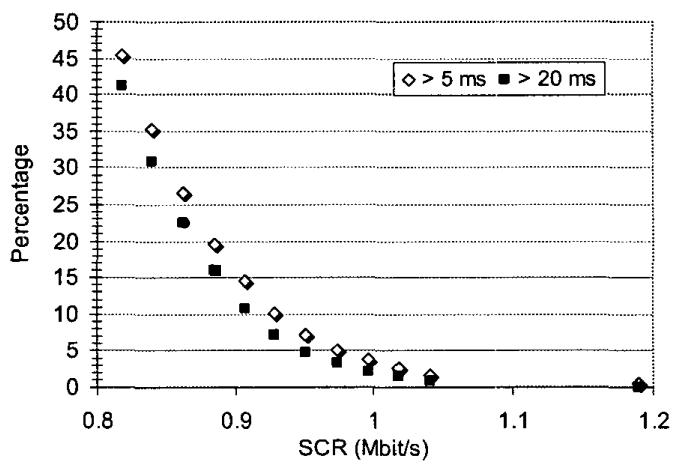

Fig. 5. Percentage of delayed web frames with rt-VBR PVP.

This kind of results may be used for the dimensioning of the shared rt-VBR PVP. The input data in this case would be the maximum allowed delay in Iub for each traffic class and the maximum percentile of the frames that may exceed that value, in order to obtain a set of "minimum" parameter values that comply with the QoS requirements of both voice and web.

\section{CONCLUSIONS AND FUTURE WORK}

In this paper we have described a simulation tool whose aim is to support the decisions related to physical topology, ATM configuration and dimensioning decisions of UMTS Iub interface. Since the operator faces many degrees of freedom, the simulator has been designed with flexibility in mind, in order to be able to model as many configurations with potential interest as possible.

We have also shown some of the actual results already obtained with this tool. At this respect we are planning to perform a wider set of simulation studies to investigate the advantages of more topologies (for instance, the benefits of having several Node-Bs in a tree or chain topology), other ATM multiplexing strategies (among them, the establishment of a separate ATM channel for each traffic class) and different traffic mixes. Besides, we also foresee to compare and refine our traffic models with actual measurements taken in a real operator's UMTS access network.

\section{ACKNOWLEDGEMENTS}

This work is funded by Telefónica Móviles España under a R\&D project inside their "program for the technological promotion of UMTS" ("Plan de Promoción Tecnológica del UMTS").

\section{REFERENCES}

[1] A. Szlovencscik, I. Godor, J. Harmatos and T. Cinkler, "Planning reliable UMTS terrestrial access networks", IEEE Communications Magazine, Vol. 40 Issue 1, pp. 6672, January 2002.

[2] B. Karlander, S. Nádas, S. Rácz and J. Reinius, "AAL2 switching in the WCDMA radio access network", Ericsson Review, Issue 3, 2002.

[3] R. Rueda, M. Gasparroni, I. Rivas and T. Kirvar, "E2E UMTS network simulator for quality of service predictions and network planning optimization", in Proceedings of XII Jornadas Telecom $1+D$, Madrid, Spain, November 2002. (In Spanish)

[4] J. Rinchiuso and K. Beshir, "Simulation of RLC for UMTS", in Proceedings of OPNETWORK 2001, Washington, D.C., USA, August 2001.

[5] R. Makke, S. Tohme, J.-Y. Cochennec and S. Pautonnier, "Performance of the AAL2 protocol within the UTRAN", in Proceedings of 2nd European Conference on Universal Multiservice Networks, Colmar, France, pp. 92-100, April 2002.

[6] A-F. Canton, S. Tohmé, D. Zeghlache and T. Chahed, "Performance analysis of AAL2/ATM in UMTS radio access network", in Proceedings of $13^{\text {th }}$ IEEE international symposium on personal, indoor and mobile radio communications, Lisboa, Portugal, September 2002.

[7] OPNET Modeler web page: http://www.opnet.com/products/modeler/

[8] O. Hersent, D. Gurle and J.-P. Petit, IP Telephony - Packetbased multimedia communications systems, AddisonWesley, 2000. (Chapter 7)

[9] 3GPP TS 34.108 V3.14.0, "Common test environments for User Equipment (UE) conformance testing (Release 1999)", December 2003.

[10]A. B. García, E. García, M. Álvarez-Campana, J. Berrocal and E. Vázquez, "A Simulation Tool for Dimensioning and Performance Evaluation of the UMTS Terrestrial Radio Access Network", Lecture Notes in Computer Science, Vol. 2515, pp. 49-60, November 2002.

[11]3GPP TR 25.933 V5.4.0, "IP transport in UTRAN (Release 5)", December 2003. (Annex A)

[12]ATM Forum, "Traffic Management Specification Version 4.1", March 1999. 